\title{
Molecular Diagnostics in Transfusion Medicine: MALDI-TOF MS for Blood Bankers- The Alternative Approach for Genotyping
}

\author{
Aus Molan \\ Department of Research and in-vitro Diagnostics/Abacus ALS/New Zealand
}

\begin{abstract}
Serology, defined as antibody-based diagnostics, has been held as the analytical gold standard in transfusion medicine. However, at the present time, the influence of molecular diagnostics in transfusion medicine is rapidly growing. These methods can improve tissue typing (HLA typing), increase safety of blood products (NAT testing of infectious diseases), and enable blood group typing in difficult circumstances (prenatal non-invasive RhD typing and/or post-transfusion of blood products). Generally speaking, molecular testing involves determining the presence of single nucleotide polymorphisms (SNPs). Blood group antigens mostly result from single nucleotide differences in critical positions. However, most blood group systems cannot be determined by looking at a single SNP. To identify members of a blood group system, a number of critical SNPs have to be considered. Current platforms used to perform molecular diagnostics are predominantly gel-based, involving time-consuming multiple manual steps. To employ molecular methods in transfusion medicine in the future, the development of higher-throughput SNP genotyping, non-gel based platforms that allow rapid, costeffective screening are essential. Because of its potential for automation, high throughput and cost effectiveness, the special focus of this review is a relatively new technique: blood group genotyping by MALDI-TOF MS. Although matrix-assisted laser desorption/ionisation, time-of-flight mass spectrometry (MALDI-TOF MS) has been previously reported for high throughput blood group genotyping, those reports are limited to only a few blood group systems. This report is timely as promising preliminary results have recently been published from a large cooperative Swiss-German project that aimed to employ MALDI-TOF MS for the molecular detection of the blood groups Rh, Kell, Kidd, Duffy, MNSs, a comprehensive collection of low incidence antigens, as well as the platelet and granulocyte antigens HPA and HNA, representing a total of 101 blood group antigens, encoded by 170 alleles, respectively. From this report, the observed success rates, data quality and concordance with known blood group types are highly impressive, underlining the accuracy and reliability of this cost-efficient high throughput method. Furthermore, Kell and RhD genotyping have been performed on foetal DNA from maternal plasma with excellent results. Additionally, current reports describe MALDI-TOF MS as a technology with short time-to-resolution, ability for high throughput, and cost efficiency when used in genetic analysis, clinical chemistry, microbiology, pharmaco-genetics, oncology and haematology. In summary, this paper examines a new technological approach for high throughput blood group genotyping by means of MALDI-TOF MS.
\end{abstract}

Keywords: MALDI-TOF MS, mass spectrometry, NAT, blood groups, blood transfusion, genome amplification technology, HLA, molecular blood genotyping, real-time PCR.

\section{INTRODUCTION}

Blood group genotyping began when Yamamoto and colleagues defined the molecular genetic basis of the histo-blood group ABO system in 1990 [1]. In 1992, Le van Kim and colleagues [2], for the first time, amplified RhD-specific polymerase chain reaction products. Typical target applications were i) genotyping apparently $\mathrm{RhD}$ negative, genetically $\mathrm{RhD}$ positive individuals, ii) detailing blood donors' antigenic profiles even from post-transfusion blood mixture samples, iii) clarifying rare blood group variants, and iv) screening for blood donors with [very] rare antigenic constellations [3-6].

Twenty years after the first genotyping attempts, more than 300 inherited blood group antigens have been described on the surface of human red cells [7], of which 284 are comprised within 30 blood group systems that have been described on a molecular level [8]. Currently, 1,419 alleles of 42 genes are listed in the Blood Group Antigen Gene Mutation Database [9]. All authenticated blood group antigens fall into one of the four ISBT classifications: systems, collections, low-incidence antigens (700 series), and high-incidence antigens (901 series) [10]. A blood group system consists of one or more antigens controlled at a single gene locus, or by two or more very closely linked homologous genes with little or no observable recombination between them [10]. Generally speaking, molecular testing involves determining the presence of single nucleotide polymorphisms (SNPs). Blood group antigens mostly result from single nucleotide differences in critical positions. However, most blood group systems cannot be determined by looking at a single SNP. To identify members of a blood group system, a number of critical SNPs have to be considered. 
Current platforms used to perform molecular diagnostics are predominantly gel-based, involving timeconsuming multiple manual steps. To employ molecular methods in transfusion medicine in the future, the development of higher-throughput SNP genotyping, non-gel based platforms that allow rapid, cost-effective screening are essential. With the emerging technology of genetic diagnosis and the genetic characterisation of polymorphisms a whole new area opens for transfusion medicine [11-13]. Being previously laborious and timeconsuming, the introduction of automation has reshaped the field of molecular characterisation. From the extraction of DNA (or RNA) to final data analysis, new instruments and software have enabled the molecular identification of large numbers of samples within limited time. These developments have greatly improved the clinical utility of molecular techniques and several reviews focusing on blood group-related genotyping approaches are forthcoming [5, 6, 14].

Matrix-assisted laser desorption/ionisation-time of flight mass spectrometry (MALDI-TOF MS) has advanced from early-stage research towards applications of real clinical relevance only within the last two decades [15]. In fact, this technology has become one of the most powerful tools for the analysis of biomolecules [16]. The first reports demonstrating successful MALDI-TOF MS biochemical analysis were published in the late 1980s from the labs of Tanaka et al. [17] and Karas and Hillenkamp [18] (Nobel prize for Chemistry to Tanaka in 2002). Subsequently, since 1991, commercial instruments have become available [19]. While MALDI-TOF MS was initially introduced in proteomics applications, Tang and colleagues demonstrated the full potential of this technique for DNA analysis in 1995 [20]. This indicates that the general utilisation of MALDI-TOF MS has emerged as an effective analytical tool to study bio-molecules only within the last 15 years. Even though a relatively young technology compared to other analytical techniques using mass spectrometry, there has been an enormous increase in the publication of MALDI-TOF MS methods and applications in the literature [5, 6, 19-21]. Three principal reasons can be attributed to this: i) the growth of proteomics as a discipline, which uses MALDI-TOF MS as a tool for post-separation protein identification; ii) the use of this high-throughput and efficient technique in applications across many life science specialisations; and iii) the promising potential of this analytical platform as a robust tool for disease biomarkers [15].

Currently, MALDI-TOF MS has well-known applications in many fields. For example, in clinical chemistry, markers for cancer [24], rheumatoid arthritis [25, 26], and Alzheimer's disease [27] can be identified with MALDI-TOF MS analysis in combination with 1-D and 2-D gel electrophoresis separations using either peptide mass fingerprinting or peptide sequence tag followed by data base searching [15]. In microbiology, MALDI-TOF MS is employed in the analysis of specific peptides or proteins directly desorbed from intact viruses, bacteria, and spores [28]. Additionally, the capability to register biomarker ions in a broad $\mathrm{m} / \mathrm{z}$ [massto-charge ratio] range, which are unique and representative for individual microorganisms, forms the basis of taxonomic identification of bacteria by MALDI-TOF MS [29]. Moreover, this technique can be applied to study either the resistance of bacteria to antibiotics or the antimicrobial compounds secreted by other bacterial species [29].

More recently, the MALDI-TOF MS method has successfully been applied to mutation screening and DNA sequencing [genotyping]. High-throughput genotyping of SNP's has the potential to become a routine method for both laboratory and clinical applications [30]. The aim of the present review is to examine an alternative method for blood group genotyping by means of a relatively new and promising molecular diagnostic technique: MALDI-TOF MS.

\section{PRINCIPLE OF OPERATION- SHINNING LIGHT ON SAMPLES}

Mass spectrometers consist of an ion source, a mass analyser that measures the mass-to-charge ratio $[\mathrm{m} / \mathrm{z}]$ of the ionised analytes, and a detector that registers the number of ions at each $\mathrm{m} / \mathrm{z}$ value. Four basic types of mass analyser are currently used in proteomic research: ion trap, time-of-flight (TOF), quadrupole and Fourier transform ion cyclotron (FT-ICR MS or FTMS) analysers. They are very different in design and performance, each with its own strength and weakness [31]. These analysers can be stand alone or, in some cases, put together in tandem to take advantage of the strengths of each. Three basic components are essential in all mass spectrometric techniques: ionisation, separation, and detection. The ionisation of an analytic molecule is the prerequisite for separation in an electromagnetic field and for the detection in an ion detector. Thus, the general principle of MS is to produce, separate, and detect gas-phase ions [16]. A focused laser beam, either in the UV or infra-red ranges, can "evaporate" compounds from the solid phase. The resulting ions are injected into a tube, accelerated, and allowed to drift towards a detector (Figure 1). MALDI uses the characteristic distribution of peptide masses obtained by chemical or enzymatic hydrolysis of proteins [32, 33]. A number of computer programs are available for using the observed peptide masses to search gene sequence databases for proteins that fit the mass fingerprint $[34,35,36]$. MALDI may be considered the fastest technique for peptide analysis [31].

Specifically, the MALDI-TOF process consists of two parts: i) laser-induced desorption/ionisation of analyte/matrix molecules (MALDI), and ii) separation and analysis of different bio-molecules based on intrinsic physical properties (TOF). A dissolved sample is mixed with a solution of an appropriate matrix and allowed to 
co-crystallise directly on special sample plates. Choosing the right matrix is essential for the success of the reaction with classic MALDI matrices being low molecular weight, organic acids like $\alpha$-cyanohydroxycinnamic acid (MW 189.17Da), 2,5-dihydroxybenzoic acid (MW 154.12Da), or 3-Hydroxypicolinic acid (MW 139.11Da) [37, 38]. The sample plate with matrix-analyte crystals is subsequently placed under a high-vacuum of the machine (pressure $<10^{-7}$ Torr) where the definite position can be controlled and monitored via video screen $[16,37]$. A nitrogen laser operating in the ultraviolet range, typically $337.1 \mathrm{~nm}$, is focused and hits the sample in pulses of 0.5-3 nanosecond durations [37, 38]. The laser energy causes structural decomposition of the irradiated crystal and generates a particle cloud [the plume] from which ions are extracted by an electric field. The mechanism behind the process of desorption may best be described as a conversion of laser energy to vibrational oscillation of the crystal molecules [16, 37]. This results in the disintegration of the crystal and the start of ion acceleration.

The acceleration in the electromagnetic field is the start of the separation process that is based on the time-of-flight principle [37, 38]. For optimal performance, the acceleration starts with a delay of 10-20 milliseconds to the ionisation (delayed extraction) [37]. After passing the grid electrode, the ions enter a fieldfree drift range where they are not accelerated further and thus travel with a speed they have reached at the moment when passing the electrode. Ion masses [mass-to-charge ratios $[\mathrm{m} / \mathrm{z}$ ] are typically calculated by measuring their TOF, which is longer for larger molecules than for smaller ones (twice the charge behaves like half the size- provided their initial energies are identical). Time-of-flight is proportional to the mass of the molecule (e.g. DNA fragment), which in turn is inversely proportional to its velocity. Arrival at the end of the flight tube is detected and recorded by a high speed recording device.

Further increase in resolution can be reached in the reflector mode (Figure 1): after having passed a distance in the drift range the ions enter another electromagnetic field and are accelerated in a nearly reversed direction towards an ion detector [37]. The reflector or ion mirror compensates for the initial energy spread of ions of the same mass coming from the ion source, and improves resolution. Ion detection is accomplished with a conversion dynode with secondary electron amplification.

The output of the detector is a time-resolved plot for each single laser pulse. After data processing, a spectrum is produced with relative intensity on the $\mathrm{y}$ axis and mass/charge on the $\mathrm{x}$ axis (Figure 2). Because predominantly single-charged, non-fragmented ions are generated, parent ion masses can easily be determined from the resulting spectrum without the need for complex data processing. The masses are accessible as numerical data for direct processing and subsequent analysis. TOFs measured during a typical MALDI experiment are in the range of several microseconds [16].

\section{MALDI-TOF MS FOR GENETIC ANALYSIS}

Recently, the MALDI-TOF MS MassARRAY compact system (Bruker Daltonics Inc., Billerica, MA) has been optimised for the detection of nucleic acids and is currently applied for SNP genotyping (including insertions and deletions), quantitative gene expression, somatic mutation screening, copy number variation analysis, and DNA methylation detection [40-44]. Because of the minimal DNA amount requirements and short amplicon length, even formalin-fixed and paraffin-embedded tissue or ancient DNA samples can be processed. Successful applications of this system in the fields of forensics, oncology, pharmacogenetics, and haematology, including $\beta$ - thalassemia genotyping have been previously reported [38, 45-48]. Additionally, MALDI-TOF MS has been successfully employed in genotyping foetal, paternally inherited SNPs [49]. Quantitative sensitivity of $5 \%$ and less has been shown [50].

In preparation for MALDI-TOF MS SNP analysis, oligonucleotides are generated using a minisequencing reaction without the need to label any nucleotides [38]. PCR is performed either in multi- or uniplex format, followed by an allele-specific single base extension of a primer that anneals directly adjacent to the SNP of interest $[38,39]$. The resulting single stranded nucleic acid analytes are deposited (nanoliter amounts) on a silicon chip (384 and 96 well formats for high throughput and low density systems, respectively) for MALDITOF analysis. The typical MassARRAY Assay workflow is illustrated in Figure 3. A major advantage of this method is that it directly measures the mass of the molecules of interest without using any proxy, such as fluorescence [52].

The time-of-flight is a specific characteristic of each DNA fragment since the $\mathrm{m} / \mathrm{z}$ ratio is an inherent property of the DNA and depends only on base composition. In the case of SNP analysis, the mass differences of the nucleotide in the polymorphic position are sufficient to discriminate an A, T, G or C (desoxyadenosine, 313Da; desoxythymidine, 304Da; desoxyguanosine, 329Da; and desoxycytosine, 289Da, respectively). These small differences in molecular mass result in different peak positions. Garritsen and colleagues [38] have demonstrated that this situation allows the discrimination between DNA fragments which differ in only one base (SNPs) and, by specific design of the size of the DNA fragments, to test multiple SNPs in one reaction. Additionally, the development of powerful software enables the automated analysis of generated MALDI-TOF MS spectra, with the possibility to assign these spectra to different genotypes. 


\section{MALDI-TOF MS IN TRANSFUSION MEDICINE}

Focusing on a set of SNPs, blood group genotyping is rapidly evolving, and several MALDI-TOF MS approached have been reported [39, 53-57]. Collaboration studies between Swiss and German researchers have successfully demonstrated the application of MALDI-TOF MS for genotyping foetal SNPs in maternal blood for non-invasive prenatal diagnosis of genetic diseases [54-56]. Moreover, they have applied this technique for the investigation of relevant SNPs for human platelet antigens (HPA1-5, HPA15) and relevant mtDNA polymorphisms in the hyper-variable regions (HV I, HV II, and HV III) $[53,56]$ in a multiplex assay. An example is shown in Figure 2. In comparison to other methods, the authors give a positive report on short timeto-resolution, ability for high throughput, and cost efficiency.

More recently however, preliminary results from a large cooperative Swiss-German project aiming to develop and implement a high through-put alternative at the Blood Transfusion Service of the Swiss Red Cross in Zurich, Switzerland, together with Sequenom GmbH, Hamburg, Germany, have been reported [39]. This project is in order to ensure the supply of rare donor blood to the Swiss population; the whole study intends to type 36,000 Swiss DNAs with the "rare module". Two thirds of the donors are expected from the Zurich area, whereas the other third may be provided by the other 12 Swiss transfusion institutions. Additional typing of HPA and HNA (human neutrophil antigen) for 3,000, and of KEL, JK, FY, MNS and RH with the presented modules for 6,000 donors will be included, correspondingly. A rough total of 1.5 million SNPs, or 125,000 multiplex reactions (326 times a 384 well plate run) will be investigated in total until the end of July 2014.

The study purpose is to employ MALDI-TOF MS for the molecular detection of the blood groups, representing a total of 101 blood group antigens, encoded by 170 alleles, respectively. The total of 170 pretyped DNAs, including rare alleles such as weak RHD type 15 (RHD*15), RHDpsi (RHD*04N.01), KEL2 (IVS3+1gNa) null (KEL*02N.06), or rare genotypes, such as KEL17 (KEL*02.17), FY*X (FY*02M.01), or IN1 (IN*01) homozygous samples, were used as positive control DNAs for verification and validation of $87 \%$ of all assays included in the various modules [39]. In case of unavailability of natural control DNAs, e.g. SC2 $(\mathrm{SC} * 02)$, LWB (LW*07), and 22 others (13\% of all assays), artificial DNA fragments were synthesised by standard PCR using one mutated and one regular amplification primer, covering the prospective diagnostic site [39].

In this study, all the blood group specificities were arranged into six modules, two specific for $\mathrm{Rh}$, and one each for Kell-Kidd-Duffy, MNSs, rare antigens, and HPA/HNA. Every module included assays for genetic $\mathrm{ABO}$, or gender determination, or both, which serve to link DNA samples to the respective donor phenotype data: crosslink ID control. According to the authors, this represents a powerful tool to exclude qualitative sample mix-up [39]. Sequenom's Assay Design software was used to select PCR primer sets that uniquely amplify only the region of interest, and validate the primers within a multiplex for unintended cross-reactions with homologous genes and other regions of the genome. All SNPs were genotyped following the standard Sequenom MassARRAY iPLEX Pro genotyping protocol and using the Sequenom MassARRAY Analyzer 4 platform. Data analysis was performed by Sequenom's TYPER 4.0 software. All the data reported below is preliminary and the current status of validation is described where appropriate; however, further validation is ongoing for all modules.

During assay verification and validation, a $100 \%$ concordance of genotyping results with pre-typed controls was observed for KEL, JK and FY Genotyping ("KEL-JK-FY module"). Among another 1,520 donor DNA genotypes, comparison with serology of KEL, Jk and Fy showed two discrepancies for Jk and one for Fy, respectively. However, erroneous serological results and novel null, or weak alleles cannot not be excluded at this stage [39].

In regards to genotyping the polygenetic "rare" low incidence blood group antigens, two multiplex reactions for rare blood group genotyping were aimed to detect a total of 34 different alleles, encoded by 24 different SNPs found on 11 different genes plus 5 crosslink ID-control specificities, three for ABO, and two for gender. In accordance with the current ISBT terminology, typing of 24 bi-allelic SNPs results in 34 different alleles, instead of 48 [39]. For example, one wild-type allele (e.g. KEL*02) simultaneously encodes several antigens (e.g., k, Kpb, Jsb, KEL11), and is therefore counted as one instead of four alleles [58]. At present, 3040 DNAs from the Zurich area have been typed using the "rare module", and rare homozygous carriers were identified for the following antigens: K $(\mathrm{n}=4)$, Kpa $(\mathrm{n}=1)$, Lua $(\mathrm{n}=5)$, LU14 $(\mathrm{n}=2)$, Ytb $(\mathrm{n}=14)$, and Coa (n $=2$ ). Observed allele frequencies in the investigated Caucasian population are concordant with those reported previously [59]. However, Gassner et al. [39] point out that beside plausibility testing of allele frequencies, direct comparisons to phenotypic results or to an independent, phenotypically validated genotyping method is mandatory for validation.

Typing for RH was done in two modules: broad and variant, with a total of five multiplex reactions and on 49 SNP positions to detect a total of 79 different $\mathrm{RH}$ alleles. Three multiplex reactions were used for the broad $\mathrm{Rh}$ antigen determination, such as $\mathrm{RhD}$ positivity and negativity, and $\mathrm{RhC/c}$, and E/e entities, plus two multiplex reactions for the detection of the variant Rh antigens, such as categories, partials, Dels, and weak D types caused by single point mutations. Gassner and colleagues [39] reported that typing on natural and artificial 
control DNAs gave the expected results for all specificities investigated. RHD exon specific SNPs represent sequence-specific positions on the RHD gene in relation to the highly homologous and generically co-amplified RHCE gene. RHD negatives of the homozygous deletion type [60] were clearly negative for all RHD exon specific SNPs. This exon screening strategy also allows for reliable RHD gene copy number (RHD zygosity) detection of every individual sample by calculating the RHD/RHCE MALDI-TOF MS peak area proportion resulting in the differentiation of RHD hetero- and homozygous individuals, at multiple genetic positions, in that order [39]. This is not only of interest in order to detect RHD zygosity, which is a difficult serological task, but also to identify RHD-CE-D hybrid alleles, masked by regular RHD genes in compound heterozygous individuals. Final RH modules were tested on $186 \mathrm{RhD}$ positive routine DNA samples. The $2 \mathrm{RH}$ modules were comparable or even more comprehensive when compared to other commercially available products. Allele coverage easily fulfils requirements for routine RHD genotyping of donors and even recipients. In addition, this system is highly customisable to individual needs due to its flexible modular design and independent assay design tools. According to Flegel [61], the Rh system is deemed to include the largest number of antigens and the most complex genetics of the 30 known blood group systems, although MNS may become a genuine competitor in the future. However, with the successful analysis of the complex RH genetics, MALDI-TOF MS genotyping has proven its principle capability as a well suited platform for molecular blood group typing.

Lastly, the HPA and HNA genotyping module for platelet and granulocyte antigen analysis allowed the parallel investigation of samples for HPA-1 to 6 and HPA-15 as well as for HNA-1 and 3-5 in one multiplex reaction. To date, all the results have been concordant with pre-typed controls. From the 1,520 platelet donor samples of the Basel and Zurich areas, HPA allele frequencies were well comparable to data previously reported for the Austrian population [62] while HNA allele frequencies are proving to be similar to those observed in Germany [63].

In addition to the modules presented above, Gassner et al. [39] are currently developing another module. It comprises detection for MN-, Ss-, Vw-, Mg-, Hut-, He-, Mt(a) antigens, and the detection of homoand heterozygous GYPB deletions, thus identifying hemi-, or homozygous U negative individuals. While all presented data is preliminary and needs further validation, the authors conclude that the observed success rates, data quality and concordance with known blood group types are highly impressive, underlining the accuracy and reliability of this cost-efficient high throughput method.

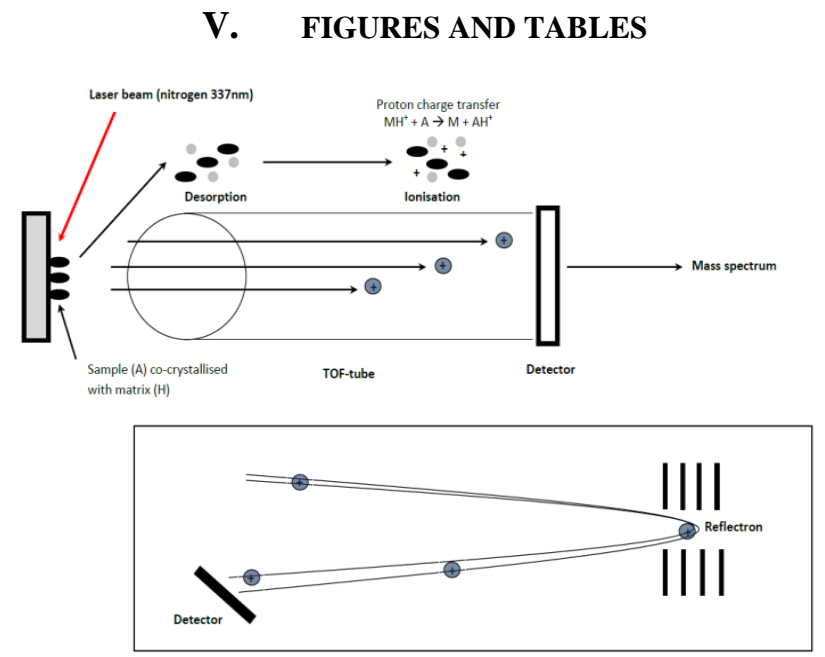

Figure 1. Schematic illustrations of linear and reflector time-of-flight MALDI MS analysis. Samples that are analysed by MALDI are first mixed with crystalline matrix and spotted on a stainless steel target. Upon drying, it is inserted into the ion source of mass spectrometer which is under a high vacuum $\left(<10^{-7}\right.$ Torr $)$. A pulsed nitrogen laser $(337 \mathrm{~nm}$ ) is fired onto the sample (ionisation of the matrix), resulting in a desorption event. The ions are repelled from the target surface and drift through the flight tube and their arrival at the detector is carefully timed, smaller ions fly faster than larger ions. Thus ions are separated according to their massdepending analyses, the ions a reflectron to resolution mass

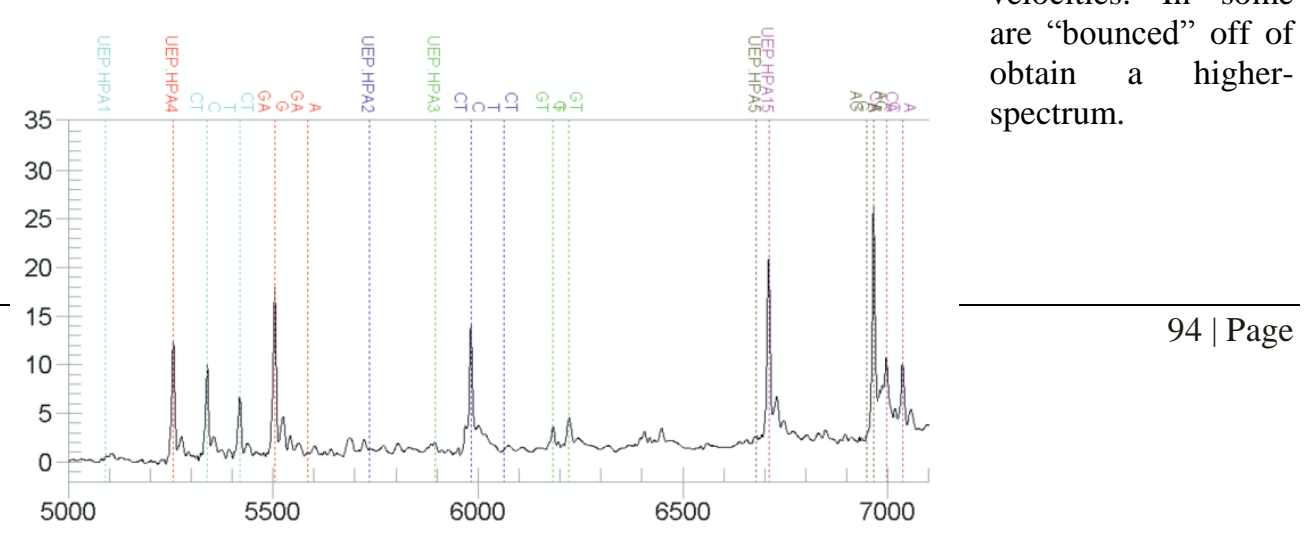


Figure 2. Mass spectra of multiplex PCR MALDI-TOF data (MassArray Iplex primer extension system) from DNA of an platelet-apheresis donor to identify relevant human platelet antigens (HPA-1 to 5 and HPA-15 are shown). The peaks corresponding to the HPA alleles are indicated with colors (Turkish blue for HPA-1, blue for HPA-2, green for HPA-3, red for HPA-4, gray for HPA-5 and pink for HPA-15). HPA genotype of this donor: HPA $1 \mathrm{a} / \mathrm{b}, 2 \mathrm{a} / \mathrm{a}, 3 \mathrm{a} / \mathrm{b}, 4 \mathrm{a} / \mathrm{a}, 5 / \mathrm{a}, 15 \mathrm{a} / \mathrm{b} . \mathrm{y}=$ relative intensity, $\mathrm{x}=\mathrm{mass}[\mathrm{m} / \mathrm{z}]$. Adapted from Garritsen et al. [38].

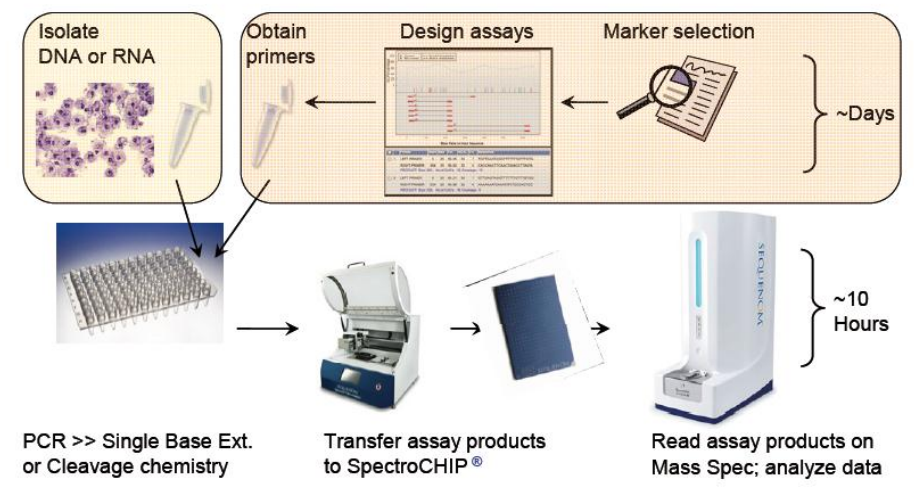

Figure 3. Typical MassARRAY Assay Workflow with final MALDI-TOF MS analysis. Adapted from Gassner et al. [51].

\section{CONCLUSION}

While potential MALDI-TOF users must consider many aspects (number of DNA's investigated, the number of SNPs these DNAs are investigated for throughput, turnaround times, requirements for donor- and recipient-specific blood group genotyping), there are many apparent advantages of MALDI-TOF. First, the small amount of sample required- in prenatal medicine, the amount of material for analysis is mostly a limiting factor. The technique proposed above uses nanoliter amounts for analysis. This minimal amount of sample underscores the potential for cost reduction. On the other hand, it requires purified samples free of ions and other impurities, increasing technical time, and sample processing costs. In addition, this method is completely automated and appropriate for high throughput analysis of as much as 384 samples running on one chip. Morover, the stability of this automated method makes it suitable as a routine testing tool for genotyping. Although the initial purchase of mass-spectrometric equipment is costly, the running costs for some particular assays, like the methods proposed from the studies of Garritsen et al. [53] and Gassner et al. [39], can be very low (3.5 cents per genotype). While the whole procedure from sample preparation to automated analysis takes 8-9 hours, there is still much development in regards to the clinical application of this technique. For SNP genotyping, a sample throughput high enough for reliable analysis can be achieved, and MALDI-TOF MS does not rely on indirect detection reactions, involving specific nucleic acid labelling or hybridisation steps. Furthermore, the MALDI-TOF platform does not need fixed formats like DNA-chips, and users are therefore free to select and customise modules of their interest. By measuring molecular mass, a direct physical property of the analyte, MALDI-TOF MS is not susceptive to background problems resulting from hybridisation-based detection reactions. In conclusion, high cost efficiency at medium levels of multiplexing, high sample throughput, and the possibility to incorporate a high level of laboratory automation will make this procedure a serious competitor in the market for SNP analysis in Transfusion Medicine.

\section{REFERENCES}

[1] F Yamamoto, H Clausen, T White, J Marken, S Hakomori. Molecular genetic basis of the histo-blood group ABO system. Nature, $345,1990,229-33$. 
[2] C Le van Kim, I Mouro, B Cherif-Zahar, V Raynal, C Cherrier, J Cartron, et al. Molecular cloning and primary structure of the human blood group RhD polypeptide. Proceedings of the National Academy of Science USA, 89, 1992, $10925-10929$.

[3] F Wagner, A Frohmajer, W Flegel. RHD positive haplotypes in D negative Europeans. BMC Genetics, 2, $2001,10$.

[4] P Rozman, T Dovc, C Gassner. Differentiation of autologous ABO, RHD, RHCE, KEL, JK, and FY blood group genotypes by analysis of peripheral blood samples of patients who have recently received multiple transfusions. Transfusion, 40, 2000, 936-942.

[5] G Denomme, S Johnson, B Pietz. Mass-scale red cell genotyping of blood donors. Transfusion and Apheresis Science, 44, 2011, 93-99.

[6] Jungbauer, C Hobel, D Schwartz, W Mayr. High-throughput multiplex PCR genotyping for 35 red blood cell antigens in blood donors. Vox Sanguinis, 102, 2012, 234-242.

[7] G Daniels, A Fletcher, G Garratty, et al. Blood group terminology 2004: from the International Society of Blood Transfusion committee on terminology for red cell surface antigens. Vox Sanguinis, 87, 2004, 304-316.

[8] J Storry, L Castilho, G Daniels, W Flegel, G Garratty, C Francis, et al. International Society of Blood Transfusion Working Party on red cell immunogenetics and blood group terminology: Berlin report. Vox Sanguinis, 101, 2011, 77-82.

[9] S Patnaik, W Helmberg, O Blumenfeld. BGMUT: NCBI dbRBC database of allelic variations of genes encoding antigens of blood group systems. Nucleic Acids Research, 40, 2012, D1023-1029.

[10] International Society Blood Transfusion ISBT. Committee on Terminology for RBC Surface Antigens. 2012 [cited 2014 January 8]. Available from: http://ibgrl.blood.co.uk/isbt\%20pages/isbt\%20terminology\%20pages/terminology\%20home\%20page.htm 2012.

[11] N Avent. Recombinant technology in transfusion medicine. Current Pharmaceutical Biotechnology, 1, 2000,117-135.

[12] W Dzik. Molecular diagnostics in transfusion medicine: the best is yet to come. Transfusion, 35, 1995,183-185.

[13] C Westhoff, S Sloan. Molecular genotyping in transfusion medicine. Clinical Chemistry, 54, 2008, $1948-1950$.

[14] D Anstee. Red cell genotyping and the future of pretransfusion testing. Blood, 114, 2009, 248-56.

[15] L Marvin, M Roberts, L Fay. Matrix-assisted laser desorption/ionization time-of-flight mass spectrometry in clinical chemistry. Clinica Chimica Acta, 337, 2003, $11-21$.

[16] C Jurinke, P Oeth, D van den Boom. MALDI-TOF Mass Spectrometry- A Versatile Tool for High-Performance DNA Analysis. Molecular Biotechnology, 26, 2004, 147-163.

[17] K Tanaka, H Waki, Y Ido, S Akita, Y Yoshida, T Yoshida. Protein and polymer analyses up to m/z 100,000 by laser ionization time-of-flight mass spectrometry. Rapid Commununications in Mass Spectrometry, 2, 1988, 151-153.

[18] M Karas, F Hillenkamp. Laser desorption ionization of proteins with molecular masses exceeding 10,000 daltons. Analytical Chemistry, 60, 1988, 2299-2301.

[19] J Stults. Matrix-assisted laser desorption/ionization mass spectrometry (MALDI-MS). Current Opinion on Structural Biology, 5, 1995, 691-698.

[20] K Tang, D Fu, S Kotter, R Cotter, C Cantor, H Koster. Matrix-assisted laser desorption/ionization mass spectrometry of immobilized duplex DNA probes. Nucleic Acids Research, 23, 1995, 3126-3131.

[21] T Bonk, A Humeny. MALDI-TOF-MS analysis of protein and DNA. Neuroscientist, 7, 2001, 6-12.

[22] D Harvey. Matrix-assisted laser desorption/ionisation mass spectrometry of oligosaccharides and glycoconjugates. Journal of Chromatography A, 720, 1996, 429-446.

[23] E Zaluzec, D Gage, J Watson. Matrix-assisted laser desorption ionization mass spectrometry: applications in peptide and protein characterization. Protein Expression and Purification, 6, 1995, 109-123.

[24] T Poon, P Johnson. Proteome analysis and its impact on the discovery of serological tumor markers. Clinica Chimica Acta, 313, 2001, 231-239.

[25] V Saulot, O Vittecoq, R Charlionet, P Fardellone, C Lange, L Marvin, et al. Presence of autoantibodies to the glycolytic enzyme alpha-enolase in sera from patients with early rheumatoid arthritis. Arthritis and Rheumatology, 46, 2002, $1196-1201$.

[26] S Blass, A Union, J Raymackers, F Schumann, U Ungethum, S Muller-Steinbach, et al. The stress protein BiP is overexpressed and is a major B and T cell target in rheumatoid arthritis. Arthritis and Rheumatology, 44, 2001, 761-71.

[27] C Nilsson, A Brinkmalm, L Minthon, K Blennow, R Ekman. Processing of neuropeptide Y, galanin, and somatostatin in the cerebrospinal fluid of patients with Alzheimer's disease and frontotemporal dementia. Peptides, 22, 2001, $2105-2112$.

[28] C Fenselau, P Demirev. Characterization of intact microorganisms by MALDI mass spectrometry. Mass Spectrometry Reviews, 20, 2001, 157-171.

[29] J Jr Lay. MALDI-TOF mass spectrometry of bacteria. Mass Spectrometry Reviews, 20, 2001, 172-194.

[30] S Sauer, I Gut. Genotyping single-nucleotide polymorphisms by matrix-assisted laser-desorption/ionization time-of-flight mass spectrometry. Journal of Chromatography, 782, 2002, 73-87.

[31] A Carpentieri. Functional and structural Protemics in apoptotic processes, doctoral diss., University of Naples Federico II, Naples, Italy, 2008.

[32] C Fini, A Amoresano, A Andolfo, S D'auria, A Floridi, S Paolini S, P Pucci. Mass spectrometry study of ecto-5'-nucleotidase from bull seminal plasma. European Journal of Biochemistry, 267, 2000, 4978-4987.

[33] S Hahner, H Ludemann, F Kirpekar, E Nordhoff, P Roepstorff, H Galla, F Hillenkamp. Matrix-assisted laser desorption/ionization mass spectrometry (MALDI) of endonuclease digests of RNA Nucleic Acids Research, 25, 1997, 1957-1964.

[34] J Adachi, C Kumar, Y Zhang, J Olsen, M Mann. The human urinary proteome contains more than 1500 proteins, including a large proportion of membrane proteins. Genome Biology, 7, 2006, R80.

[35] K Parker. Scoring methods in MALDI peptide mass fingerprinting: ChemScore, and the ChemApplex program. Journal of the American Society of Mass Spectrometry, 13, 2002, 22-39.

[36] C Sutton, K Pemberton, J Cottrell, J Corbett, C Wheeler, M Dunn. Identification of myocardial proteins from two-dimensional gels by peptide mass fingerprinting. Electrophoresis, 16, 1995, 308-316.

[37] Pappin AnagnosTec. Technology - MALDI-TOF MS principles. No date [cited 2014 January 8]. Available from: http://www.anagnostec.eu/maldi-tof-ms/technology.html

[38] H Garritsen, A Fan, D Lenz, H Hannig, Z Zhong, R Geffers, W Lindenmaier, K Dittmar, B Wörmann. Molecular Diagnostics in Transfusion Medicine: In Capillary, on a Chip, in Silico, or in Flight? Transfusion Medicine and Hemotherapy, 36, 2009 , $181-187$.

[39] C Gassner, S Meyer, B Frey, C Vollmert. Matrix-Assisted Laser Desorption/Ionisation, Time-of-Flight Mass Spectrometry-Based Blood Group Genotyping - The Alternative Approach. Transfusion Medicine Reviews, 27, 2013, 2-9.

[40] M Millis. Medium-throughput SNP, genotyping using mass spectrometry: multiplex SNP genotyping using the iPLEX(R) Gold assay. Methods in Molecular Biology, 700, 2011, 61-76.

[41] D Fumagalli, P Gavin, Y Taniyama, S Kim, H Choi, S Paik, et al. A rapid, sensitive, reproducible and cost-effective method for mutation profiling of colon cancer and metastatic lymph nodes. BMC Cancer, 10, 2010, 101.

[42] S Docherty, O Davis, C Haworth, R Plomin, J Mill. DNA methylation profiling using bisulfite-based epityping of pooled genomic DNA. Methods, 52, 2010, 255-258. 
[43] M Bradic, J Costa, I Chelo. Genotyping with Sequenom. Methods in Molecular Biology, 772, 2011, 193-210.

[44] K Meyer, P Ueland. Use of matrix-assisted laser desorption/ionization time-of-flight mass spectrometry for multiplex genotyping. Advancements in Clinical Chemotherapy, 53, 2011, 1-29.

[45] M Jaremko, C Justenhoven, B Abraham, W Schroth, P Fritz, S Brod S, et al. MALDITOF MS and TaqMan assisted SNP genotyping of DNA isolated from formalin-fixedand paraffin-embedded tissues (FFPET). Human Mutation, 25, $2005,232-238$.

[46] F Mendisco, C Keyser, C Hollard, V Seldes, A Nielsen, E Crubezy, et al. Application of the iPLEX(TM) Gold SNP genotyping method for the analysis of Amerindian ancient DNA samples: Benefits for ancient population studies. Electrophoresis, 32, 2011, 386-93.

[47] M Looi, M Sivalingam, N Husin, F Radin, R Isa, S Zakaria, et al. Multiplexed genotyping of beta globin mutations with MALDITOF mass spectrometry. Clin Chimica Acta, 412, 2011, 999-1002.

[48] H Liao, Y Su, H Kao, C Hung, H Wang, Y Chen. Parallel minisequencing followed by multiplex matrix-assisted laser desorption/ionization mass spectrometry assay for beta-thalassemia mutations. Journal of Human Genetics, 50, 2005, 139-50.

[49] Li, F Wenzel, W Holzgreve, S Hahn. Genotyping fetal paternally inherited SNPs by MALDI-TOF MS using cell-free fetal DNA in maternal plasma: influence of size fractionation. Electrophoresis, 27, 2006, 3889-3896.

[50] A Vivante, N Amariglio, M Koren-Michowitz, O Ashur-Fabian, G Nagler, G Rechavi, et al. High-throughput, sensitive and quantitative assay for the detection of BCRABL kinase domain mutations. Leukemia, 21, 2007, 1318-1321.

[51] C Gassner, M Frey, C Vollmert. Report on a High-throughput Blood Group Genotyping Prototype Using Matrix-assisted Laser Desorption /Ionization Time-of-Flight Mass Spectrometry (MALDI-TOF MS). Poster session presented at: 64th American Association of Blood Banks Annual Meeting, AABB \& CTTXPO 2011, San Diego, CA, USA.

[52] J Ragoussis, G Elvidge, K Kaur, S Colella. Matrix-assisted laser desorption/ionisation, time-of-flight mass spectrometry in genomics research. PLoS Genetics, 2, 2006, e100.

[53] H Garritsen, A Fan, N Bosse, H Hannig, R Kelsch, H Kroll, W Holzgreve, X Zhong. Matrix-assisted laser desorption/ionization time-of-flight mass spectrometry for genotyping of human platelet- specific antigens. Transfusion, 49, 2009, $252-258$.

[54] Y Li, K Finning, G Daniels, S Hahn, X Zhong, W Holzgreve. Noninvasive genotyping fetal Kell blood group (KEL1) using cellfree fetal DNA in maternal plasma by MALDI-TOF mass spectrometry. Prenatal Diagnosis, 28, 2008, $203-208$.

[55] Y Li, S Hahn, W Holzgreve. Recent developments in the detection of fetal single gene differences in maternal plasma and the role of size fractionation. Annals of the New York Academy of Sciences,1092, 2006, 285-292.

[56] Y Li, F Wenzel, W Holzgreve, S Hahn. Genotyping fetal paternally inherited SNPs by MALDI-TOF MS using cell-free fetal DNA in maternal plasma: influence of size fractionation. Electrophoresis, 27, 2006, 3889-3896.

[57] F Xiu-Cheng, H Garritsen, S Tarhouny, M Morris, S Hahn, W Holzgreve, X Zhong. A rapid and accurate approach to identify single nucleotide polymorphisms of mitochondrial DNA using MALDI-TOF mass spectrometry. Clinical Chemistry and Laboratory Medicine, 46, 2008, 299-305.

[58] International Society Blood Transfusion ISBT. Terminology for blood group alleles. 2012 [cited 2014 February 8]. Available from: http://ibgrl.blood.co.uk/ISBTPages/AlleleTerminology/Allele-Terminology.htm

[59] G Daniels. Human Blood Groups. 2nd ed. Oxford: Blackwell Science; 2002.

[60] F Wagner, W Flegel. RHD gene deletion occurred in the Rhesus box. Blood, 95, 2000, 3662-3668.

[61] W Flegel. Molecular genetics and clinical applications for RH. Transfusion Apheresis Science, 44, 2011, 81-91.

[62] Immuno-Polymorphism-Database. IPD - HPA Database. 2013 [cited 2014 Febuary 8]. Available from: http://www.ebi.ac.uk/ipd/hpa/freqs_1.html

[63] B Hauck, A Philipp, R Eckstein, S Ott, R Zimmermann, T Dengler, et al. Human neutrophil alloantigen genotype frequencies among blood donors with Turkish and German descent. Tissue Antigens, 78, 2011, 416-20. 